\title{
Diverse Facets of Sphingolipid Involvement in Bacterial Infections
}

\author{
Tobias C. Kunz and Vera Kozjak-Pavlovic* \\ Department of Microbiology, Biocenter, University of Würzburg, Würzburg, Germany
}

Sphingolipids are constituents of the cell membrane that perform various tasks as structural elements and signaling molecules, in addition to regulating many important cellular processes, such as apoptosis and autophagy. In recent years, it has become increasingly clear that sphingolipids and sphingolipid signaling play a vital role in infection processes. In many cases the attachment and uptake of pathogenic bacteria, as well as bacterial development and survival within the host cell depend on sphingolipids. In addition, sphingolipids can serve as antimicrobials, inhibiting bacterial growth and formation of biofilms. This review will give an overview of our current information about these various aspects of sphingolipid involvement in bacterial infections.

\section{OPEN ACCESS}

Edited by:

Burkhard Kleuser,

University of Potsdam, Germany

Reviewed by:

Rodrigo F. M. De Almeida,

University of Lisbon, Portugal

Luis M. S. Loura,

University of Coimbra, Portugal

Pedro Escoll,

Institut Pasteur, France

*Correspondence:

Vera Kozjak-Pavlovic

vera.kozjak@uni-wuerzburg.de

Specialty section:

This article was submitted to Membrane Physiology and Membrane Biophysics,

a section of the journal

Frontiers in Cell and Developmental

Biology

Received: 28 June 2019 Accepted: 05 September 2019 Published: 19 September 2019

Citation:

Kunz TC and Kozjak-Pavlovic V (2019) Diverse Facets of Sphingolipid

Involvement in Bacterial Infections.

Front. Cell Dev. Biol. 7:203.

doi: 10.3389/fcell.2019.00203
Keywords: infection, pathogenic bacteria, sphingolipids, ceramide, autophagy

\section{INTRODUCTION}

Sphingolipids belong to a class of lipids defined by their amino-alcohol backbone. They were considered merely to be ubiquitous components of the eukaryotic cell membrane, shown to play a critical role in the formation of membrane microdomains called lipid rafts that are important for cell signaling (Simons and Ikonen, 1997). However, in the past decades, it has been revealed that many sphingolipids are bioactive lipids that regulate a large subset of cellular functions, such as apoptosis or autophagy (Cuvillier et al., 1996; Harvald et al., 2015). Although sphingolipids vary greatly in their structure and function, their synthesis and degradation are mediated by common synthetic and catabolic pathways. Sphingolipids can be synthesized de novo, by the hydrolysis of sphingomyelin, or through the salvage pathway, by recovery of sphingosine from complex sphingolipids (Figure 1). In all cases the result is the synthesis of ceramide, which represents the starting point for the creation of complex sphingolipids and thus is involved in many regulating processes within the cell. The metabolism of sphingolipids has been extensively reviewed by Gault et al. (2010). Ceramide itself regulates growth and development and promotes cell survival and division (Mencarelli and Martinez-Martinez, 2013).

The de novo biosynthesis of ceramide starts at the endoplasmic reticulum (ER) with an enzyme called serine palmitoyltransferase. This enzyme catalyzes the condensation of serine and fatty acid-CoA to 3-ketosphinganine. Afterward, 3-ketosphinganine gets reduced by 3-ketosphinganine reductase and then is processed by dihydroceramide synthase to dihydroceramide. In the final step of the de novo ceramide biosynthesis, a dihydroceramide desaturase introduces a double bond to create ceramide (Perry, 2002; Menaldino et al., 2003; Figure 1).

Constitutive degradation of sphingolipids and glycosphingolipids takes place in the late endosomes and lysosomes at acidic pH to form sphingosine (Riboni et al., 1997; Kolter and Sandhoff, 2005). The oligosaccharide chains of glycosphingolipids are stepwise removed by the release of monosaccharide units through exohydrolases. In the salvage pathway, long-chain sphingoid bases are broken down to sphingosine, which is reacylated to form ceramide by an 


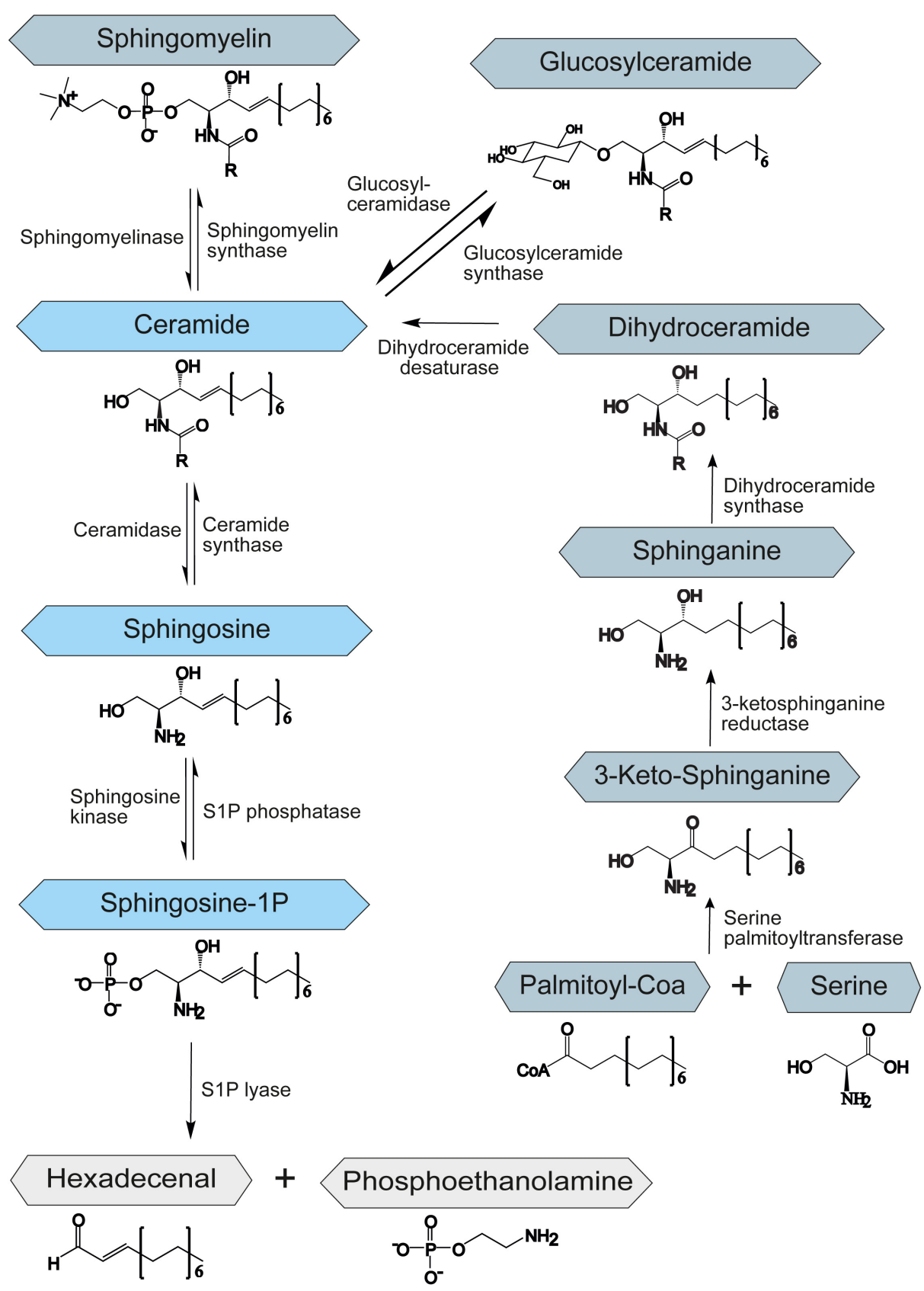

FIGURE 1 | Schematic representation of the sphingolipid metabolism. S1P - sphingosine-1-phosphate. The central role of the sphingolipid metabolism plays ceramide. It is synthesized de novo from serine and palmitoyl-CoA, by hydrolysis of sphingomyelin or through the salvage pathway by recovery of sphingosine from complex sphingolipids. Degradation of sphingolipids occurs through the degradation of S1P to hexadecenal and phosphoethanolamine by an enzyme called S1P-lyase.

enzyme called ceramide synthase. Thus, ceramide synthase family members probably trap free sphingosine released from the lysosome at the surface of the ER or in ER-associated membranes. The salvage pathway is estimated to contribute to $50-90 \%$ of sphingolipid biosynthesis (Gillard et al., 1998; Tettamanti et al., 2003).

During the generation of ceramide from sphingosine, ceramide synthases add different fatty acyl chains at the
C2-amino group of the sphingosine backbone, resulting in numerous and diverse sphingolipids. Variations in the chain length of ceramide acyl chains are linked to potentially altered membrane bilayer dynamics or differential signaling properties by recruitment of different binding partners. This topic has previously been reviewed by Grösch et al. (2012). However, the effect of different length of acyl chains on ceramide properties and function is poorly understood and needs further investigation. 
Ceramide exerts a specific function in mitochondria, where the increase in ceramide has been linked to the induction of apoptosis. Ceramide pool in mitochondria seems to be regulated by the localized activity of ceramide synthase, sphingomyelinases, and neutral ceramidase. However, another source of mitochondrial ceramide could be the ER, due to the proximity and close interaction of these two organelles (Hernández-Corbacho et al., 2017).

Apart from ceramide, sphingosine-1-phosphate (S1P) has been shown to be a potent signaling molecule. It is linked to the regulation of mitochondrial function (Bajwa et al., 2015), gene expression (Davaille et al., 2000), and ER stress (Lépine et al., 2011). Moreover, it was implicated in the regulation of important processes such as apoptosis, autophagy, and cell proliferation (Harvald et al., 2015). S1P is synthesized by the sphingosine kinase-1 and -2 (SPHK1/2) by phosphorylation of sphingosine and degraded by the S1P phosphatase (SGPP) or lyase (SGPL1) to sphingosine or hexadecenal and phosphoethanolamine, respectively (Figure 1). While SPHK1 is mainly associated with cell survival (Sarkar et al., 2005), SPHK2 has been shown to influence mitochondrial function and homeostasis. It has also been involved in regulation of histone deacetylases and thereby in suppression of cell growth and promotion of apoptosis (Liu et al., 2003). SGPP and SGPL1 ensure balanced levels of S1P and other sphingolipid intermediates that may control cell growth and death. The upregulation of SGPL1 results in an accumulation of hexadecenal, which was shown to be cytotoxic (James and Zoeller, 1997).

In the last years, sphingolipids have been revealed as key players in infection processes. Even though most pathogenic bacteria do not produce their own sphingolipids, they are capable of utilizing or degrading host sphingolipids to promote their virulence. This review will summarize some of our current knowledge about involvement of sphingolipids in bacterial infection, starting from the interaction with pathogenic bacteria on the surface of the cell, including the uptake of bacteria, immune response, survival and propagation of intracellular bacteria, and ending with several remarks on the bactericidal effects of sphingolipids.

\section{BACTERIAL ENTRY - ROLE OF SPHINGOLIPIDS IN BACTERIAL ADHESION AND UPTAKE}

Glycosphingolipids frequently serve as receptors for Escherichia coli and other bacteria such as Pseudomonas aeruginosa, Bordetella pertussis, Mycoplasma pneumoniae, and Helicobacter pylori [reviewed in Hanada (2005)]. A prominent example is GM1 ganglioside, which serves as a receptor for cholera toxin (Holmgren et al., 1975). Also, ceramide-enriched lipid rafts acting as binding platforms, as well as sphingolipid signaling, such as through the activation of acid sphingomyelinase (ASM) (Simonis and Schubert-Unkmeir, 2018), often mediate the entry of bacterial pathogens into host cells, which is a step important for infection and establishment of bacteria in an intracellular niche (Figure 2).
For pathogenic Neisseria, sphingolipids play an important role in the adhesion and invasion into the host cell. Neisseria meningitidis, a causative agent of meningitis and meningococcal sepsis, similar to Haemophilus influenzae binds to specific glycosphingolipids on the host cell surface, which can be found on human granulocytes and oropharyngeal epithelium, the preferential habitat for these two pathogens (Hugosson et al., 1998). Neisseria express highly variable lipooligosaccharides (LOS) on their surface, molecular mimics of glycosphingolipids found on human cells. LOS undergo phase variation, which is important for immune evasion, as well as adherence and invasion (Harvey et al., 2001).

Several reports emphasize the role of sphingomyelinases in the invasion of pathogenic Neisseria. The activation of ASM by $N$. gonorrhoeae, a bacterium causing sexually transmitted disease gonorrhea, is important for the entry of these bacteria into nonphagocytic cells (Grassmé et al., 1997), which occurs through the Opa-mediated interaction of bacteria with carcinoembryonic antigen-related cell adhesion molecule (CEACAM) receptors (Hauck et al., 2000). Neutral sphingomyelinase (NSM) also plays a role in the uptake of $N$. gonorrhoeae (Faulstich et al., 2015), but by a different mechanism, the so-called $\operatorname{PorB}_{I A^{-}}$ mediated low phosphate-dependent invasion (Kühlewein et al., 2006). N. meningitidis likewise causes the activation of ASM and ceramide release that are essential for the internalization of meningococci into brain endothelial cells, which is connected to the expression of the outer membrane protein OpcA and binding to cell surface heparan sulfate proteoglycans (HSPGs) (Simonis et al., 2014). Recently, a role of meningococcal pilus in the translocation of ASM to the surface of infected cells has been described (Peters et al., 2019). In all cases, invasion of the host cell contributes to immune evasion and spreading of these pathogenic bacteria from the site of the initial contact to other tissues.

Uptake of bacteria through activation of sphingomyelinases as seen for Neisseria is not always beneficial for the pathogen but represents a host defense mechanism, as well. For example, $P$. aeruginosa, associated with serious hospital-acquired and opportunistic infections, activates host ASM, leading to generation of plasma membrane ceramide-enriched platforms that mediate the internalization of bacteria, apoptosis induction and cytokine response (Grassme et al., 2003). In macrophages, as well as in neutrophils, infection with $P$. aeruginosa leads to cell death, which is important for the clearing of infection. In both cases the role of sphingomyelinases in this process has been proposed. For alveolar macrophages, after the activation of the ASM upon infection, the formation of ceramide-enriched platforms serves to amplify ASM-mediated redox signaling, which eventually leads to macrophage apoptosis (Zhang et al., 2008). In the case of neutrophils, the apoptosis is mediated through pyocyanin, a pigment and toxin released by $P$. aeruginosa, and through mitochondrial ASM (Managò et al., 2015). However, $P$. aeruginosa can possibly counteract the increase in ceramide through secretion of hemolytic phospholipase $\mathrm{C}$ that can synthesize sphingomyelin from ceramide, and alkaline ceramidase, which can break down 


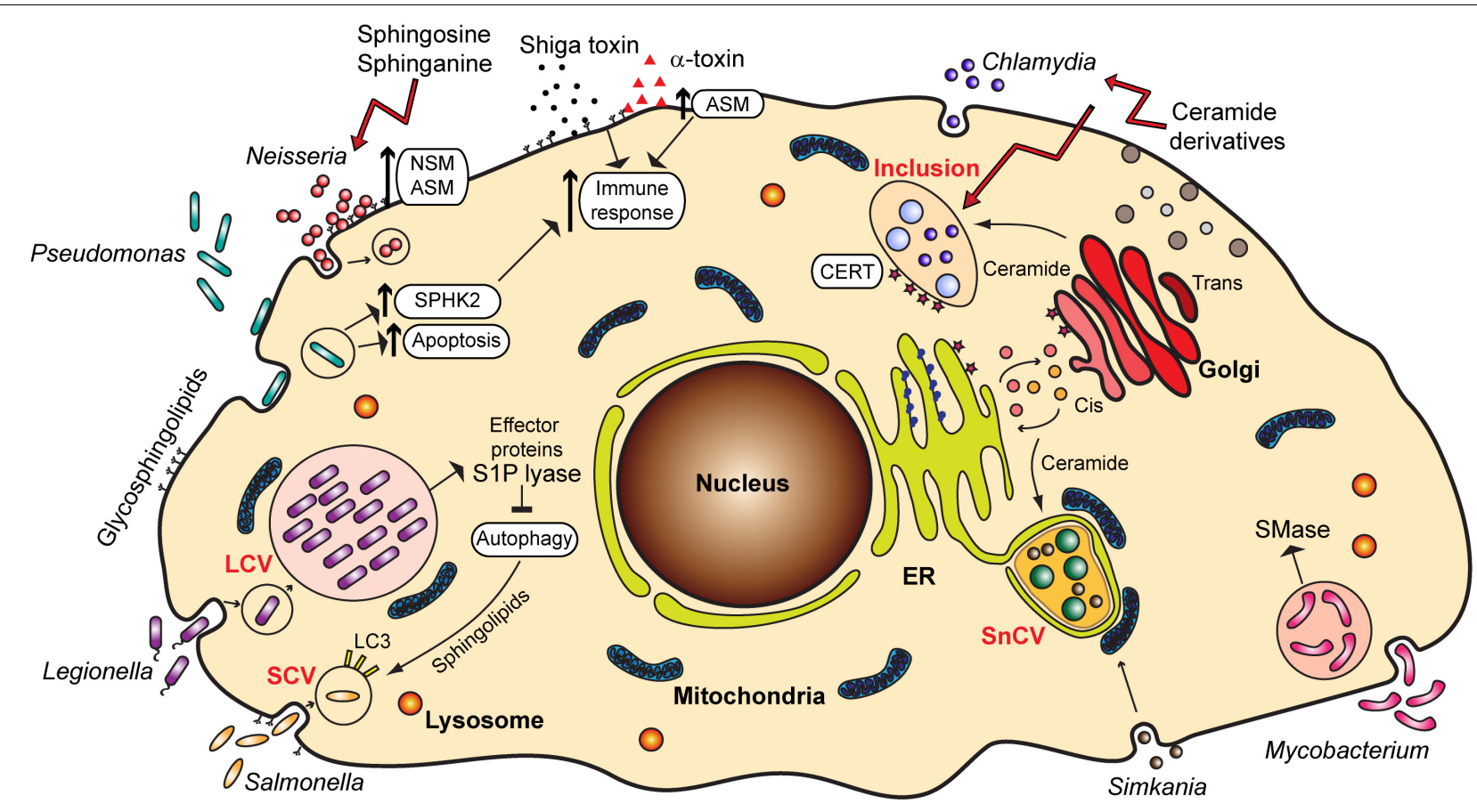

FIGURE 2 | Overview of the various bacterial pathogens and their interaction with sphingolipids and sphingolipid signaling pathways. ER, endoplasmic reticulum; LCV, Legionella-containing vacuole; SCV, Salmonella-containing vacuole; SnCV, Simkania negevensis-containing vacuole; ASM, acid sphingomyelinase; NSM, neutral sphingomyelinase; SPHK2, sphingosine kinase 2; S1P, sphingosine-1-phosphate; SMase, sphingomyelinase; CERT, ceramide transfer protein; LC3, Microtubule-associated protein 1A/1B-light chain 3.

ceramide to avoid generation of ceramide-enriched platforms and uptake, and increase hemolysis (Okino et al., 1998; Okino and Ito, 2007).

Not only ceramide, but sphingosine and S1P play a part in lung inflammatory injury caused by $P$. aeruginosa. SPHK2 is phosphorylated upon $P$. aeruginosa infection, which increases its nuclear localization, leading to higher levels of S1P and increased histone acetylation. This as a consequence has an enhanced expression of pro-inflammatory genes and secretion of pro-inflammatory cytokines, interleukin-6, and tumor necrosis factor- $\alpha$ (Ebenezer et al., 2019). Lastly, surface sphingolipids are also involved in infection with $P$. aeruginosa. The bacterial pilin binds to asialo GM1, but not to sialylated gangliosides. Considering that the surface of epithelial cells from cystic fibrosis patients shows differences in sialylation of glycolipids, this might enhance susceptibility of cystic fibrosis patients to infections with P. aeruginosa (Saiman and Prince, 1993).

Sphingolipids seem also to play a specific role in the internalization of Mycobacteria, among which are the causative agents of tuberculosis and leprosy, by macrophages, as shown for Mycobacterium smegmatis. For this bacterium, the metabolic depletion of sphingolipids in J774a.1 macrophages led to a decrease in entry of bacteria into the host cells (Viswanathan et al., 2018).

Finally, the pathogenicity of bacteria is often mediated by the binding of bacterial toxins to gangliosides and glycosphingolipids, but this aspect of the role of sphingolipids in bacterial infections will not be reviewed here in detail. Prominent examples are Shiga toxin from Shigella dysenteriae [reviewed in Kavaliauskiene et al. (2017)] and Staphylococcus aureus and its $\alpha$-toxin. $\alpha$-toxin has been reported to activate ASM, which leads to changes in permeability and lung edema (Becker et al., 2017). Ceramide increase upon $S$. aureus infection also stimulates pro-inflammatory signaling through a release of cathepsin $\mathrm{B}$ and D from lysosomes (Ma et al., 2017; Figure 2). Therefore, the inflammation, edema and lung tissue damage upon $S$. aureus infection seem to be induced by the binding of $\alpha$-toxin and activation of ASM.

Inhibition of sphingomyelinases as a means of controlling inflammation and spread of infection might be consequently beneficial for S. aureus infection (Peng et al., 2015), or infection with pathogenic Neisseria, but is detrimental in case of infection with $P$. aeruginosa (Grassme et al., 2003). It is therefore of great importance to understand the mechanisms underlying infections with different pathogenic bacteria, so that this knowledge could be translated to appropriate treatments.

\section{ONCE INSIDE - ROLE OF SPHINGOLIPIDS IN BACTERIAL REPRODUCTION AND SURVIVAL}

Uptake of bacteria into cells can be a mechanism for bacterial killing, through formation of phagolysosomes or induction of autophagy. However, bacteria can avoid death by escaping into the cytosol, preventing fusion of phagosomes and lysosomes, 
blocking autophagy, or, in some cases, surviving within the lysosomal environment. Certain bacteria also reside in nonlysosomal compartments inside the cell where they multiply, avoiding recognition and immune response. Sphingolipids have important function in many of these strategies for the survival of pathogenic bacteria within the host cell.

\section{Sphingolipids in Bacterial Development and Reproduction}

Obligate intracellular bacteria heavily depend on the host cell for the procurement of the metabolites required for their development. Sphingolipids represent important building blocks for the membranes of compartments in which these bacteria reside and multiply (Figure 2).

Chlamydia trachomatis, a causative agent of trachoma and sexually transmitted disease, is characterized by a biphasic life cycle, in which a reproductive part is spent inside the host cell, within a vesicular compartment known as an inclusion. Chlamydia directly obtain ceramide from the host cell Golgi and incorporate it into the inclusion membrane (Hackstadt et al., 1995; Figure 2). This is a process essential for the pathogen survival, in which chlamydial inclusion fuses with trans-Golgi network-derived secretory vesicles (van Ooij et al., 2000) in an Akt and Rab14-mediated way (Capmany and Damiani, 2010; Capmany et al., 2019). C. trachomatis has also been reported to cause the fragmentation of Golgi and to induce formation of mini stacks in the vicinity of the inclusion membrane, which supports lipid acquisition from the host (Heuer et al., 2009). Interestingly, it is possible that C. trachomatis establishes a sphingomyelin biosynthetic factory at or near the inclusion with the help of host cell proteins. Moreover, Elwell et al. (2011) showed that $C$. trachomatis co-opts Golgi-specific Brefeldin A resistance guanine nucleotide exchange factor 1 (GBF1), a protein important for the assembly and maintenance of the Golgi stack, for sphingomyelin acquisition contributing to the growth and stability of the inclusion. For the replication, however, C. trachomatis recruits ceramide transfer protein (CERT), vesicleassociated membrane protein-associated protein A (VAP-A), and sphingomyelin synthases, SMS1 and SMS2 to the inclusion membrane, therewith obtaining ceramide and converting it into sphingomyelin close to the inclusion (Elwell et al., 2011). Recruitment of CERT occurs with the help of chlamydial effector protein IncD present in the inclusion membrane (Agaisse and Derre, 2014). Other reports implicate additionally the Src family tyrosine kinase Fyn (Mital and Hackstadt, 2011), as well as trans-Golgi SNARE protein syntaxin 6 (Moore et al., 2011) in sphingolipid trafficking to chlamydial inclusion.

The described acquisition of sphingolipids from the host cells seems to be a common occurrence among chlamydia. Both Chlamydia pneumoniae and Chlamydia psittaci follow similar mechanisms, involving exocytic vesicles or CERT protein (Wolf and Hackstadt, 2001; Koch-Edelmann et al., 2017). In addition, a chlamydia-like microorganism Simkania negevensis has been likewise reported to obtain ceramide from the host cell, a process most likely dependent on the retrograde transport (Herweg et al., 2016).
Mycobacterium tuberculosis, although not strictly intracellular, uses host macrophages and dendritic cells for replication. During this process, the mycobacteria have been shown to depend on sphingomyelin. M. tuberculosis express the protein Rv0888, which exhibits a sphingomyelinase activity and cleaves host sphingomyelin into ceramide and phosphorylcholine. These compounds are used by bacteria as the sources of carbon, nitrogen and phosphorus (Speer et al., 2015). However, the purpose of $M$. tuberculosis-produced sphingomyelinases might be not only to provide a source of nutrients, but to modulate sphingolipid signaling and cell death induction, thereby controlling the immune response to this pathogen (Castro-Garza et al., 2016).

\section{Sphingolipid Importance for the Survival of Intracellular Bacteria}

Autophagy and apoptosis play a crucial role in controlling infection with various bacteria and viruses, representing an important part of innate immunity, but also being manipulated occasionally by pathogens for the purpose of survival or replication (Rudel et al., 2010; Siqueira et al., 2018). Sphingolipids were shown to be involved in the regulation of both apoptosis and autophagy, with ceramide being associated with cell death and S1P promoting cell survival (Young et al., 2013). Increase in intracellular levels of ceramide or treatment with sphingomyelinase has been reported to induce apoptosis in HL-60 or U937 cells, an effect which could be prevented by exposure of the cells to S1P (Cuvillier et al., 1996). For this, the term "sphingolipid rheostat" has been introduced to describe regulation of cell fate through the interconversion between ceramide and S1P. However, a multitude of later studies has shown a great complexity of the signaling mechanisms by which these sphingolipid metabolites influence cell death (Newton et al., 2015). As bacteria often modulate apoptosis to accommodate the host cell to their own needs (Rudel et al., 2010) it is possible that this modulation partially takes place through sphingolipid signaling, and that mitochondrial sphingolipids play a special role in this process. This direction is certainly worth exploring in the future, especially for intracellular bacteria.

In addition to apoptosis, autophagy and the associated cell death have also been subject to regulation by "sphingolipid rheostat" (Taniguchi et al., 2012; Young et al., 2013). Autophagy is a highly conserved catabolic process through which unnecessary or damaged components are degraded to maintain cellular homeostasis (Young et al., 2013; Young and Wang, 2018). In brief: a cargo is engulfed by a membrane forming the socalled autophagosome. Mature autophagosomes can then fuse with lysosomes, leading to the degradation of the cargo (Miller and Celli, 2016). It has been shown that S1P upregulates autophagy, therefore promoting cell survival, although several reports indicated that S1P can also act as an inhibitor of autophagy through activation of the mammalian target of rapamycin (mTOR) (Harvald et al., 2015). Ceramide induces autophagy and mitophagy, could be important for fusion of autophagosomes with lysosomes, and is involved in induction of autophagic cell death. In addition, the "many ceramides" 
hypothesis implies that the function of ceramide depends on the chain length, adding a level of complexity to the regulation of autophagy through sphingolipids (Young et al., 2013; Harvald et al., 2015). Autophagy can be further subdivided in selective and non-selective. Non-selective autophagy describes the degradation of a random portion of a cytosol to provide nutrients during starvation or to degrade long-lived proteins. Selective autophagy targets specific cellular compartments. This includes utilizing the autophagy machinery for the removal of mitochondria (mitophagy) or the clearance of intracellular pathogens (xenophagy) (Deretic et al., 2013; Huang and Brumell, 2014). Hence, evading or regulating autophagy is important for the pathogen survival.

One well-studied pathogen regulating the autophagic machinery is Legionella pneumophila, a gram-negative bacterium naturally replicating in protists in aquatic environments. It is the causative agent of the Legionnaires disease (Newton et al., 2010). As many signaling pathways are conserved in human macrophages and protists, L. pneumophila are capable of invading human cells, where they replicate in vacuoles, the so-called Legionella-containing vacuole (LCV). Inside the cell, L. pneumophila secretes over 300 effector proteins interfering with a broad range of cellular pathways. It has been shown that many of these effector proteins have a structure similar to eukaryotic proteins that are never or rarely found in prokaryotic genomes. It is hypothesized that L. pneumophila acquired these proteins through horizontal gene transfer from its host (Cazalet et al., 2004; Gomez-Valero and Buchrieser, 2013). Among those proteins, three proteins share similarities to eukaryotic proteins of the sphingolipid pathway: Lpp2641 (putative sphingomyelinase), Lpp2295 (putative sphingosine kinase) and Lpp2128 or LpSPL (S1P lyase) (Rolando et al., 2016a). The latter, being a S1P lyase, leads to the degradation of S1P, which is a critical mediator for controlling the balance between sphingolipid-induced autophagy and cell death. Moreover, macrophages infected with L. pneumophila show an overall reduction of bioactive sphingolipids. Macrophages infected with an LPSPL mutant strain show increased levels of sphingosine compared to wildtype L. pneumophila and indeed, $L p S P L$ was confirmed to restrain autophagy by acting on autophagosome biogenesis. Thus, L. pneumophila actively modulates sphingolipid metabolism to evade the cell autophagic response (Rolando et al., 2016b; Figure 2).

Another pathogen indicated to manipulate autophagy via sphingolipids is Salmonella enterica, an intestinal pathogen that represents a major public health threat due to increasing antibiotic resistance. Similar to Legionella, Salmonella forms a vacuole, called the Salmonella-containing vacuole (SCV). A recent publication extensively reviews the importance of sphingolipids for Salmonella infection from adherence to clearance, discussing the interaction of Salmonella, sphingolipids and the autophagic machinery (Huang, 2017).

Salmonella has been shown to activate the focal adhesion kinase and to recruit it to the SCV. This kinase promotes the activation of the Akt pathway and consequently mTOR is stimulated. This results in the suppression of autophagy and bacterial survival (Owen et al., 2014). More recent studies demonstrate that Salmonella does not only inhibit but actively regulates the autophagic pathway. In the early stages of infection, Salmonella triggers the amino acid starvation and mTOR inhibition, resulting in the induction of autophagy (Tattoli et al., 2012). However, in later stages the increase of cytosolic amino acid levels in infected cells reactivates mTOR at the surface of the SCV (Narayanan and Edelmann, 2014; Figure 2).

Inhibition of Akt signaling as well as the activation of extracellular signal-regulated kinase (ERK) $1 / 2$ activity is associated with autophagy in colon adenocarcinoma cells (e.g., HT29 and HCT-15) (Ogier-Denis et al., 2000; Kanazawa et al., 2004; Ellington et al., 2006). In this regard, activated ERK was shown to upregulate beclin-1 expression, resulting in the induction of autophagy (Liu et al., 2012). Inhibition of the de novo biosynthesis of sphingolipids by myriocin, an inhibitor of the enzyme serine palmitoyl transferase, leads to decreased autophagy through the activation of Akt and downregulation of beclin-1 (Scarlatti et al., 2004). Moreover, myriocin downregulates ERK and represses the membrane recruitment of NOD2 and ATG16L1. The interaction of ATG16L1 and NOD2 in epithelial cells leads to autophagic degradation of Salmonella. In addition, myriocin decreased the Salmonella-induced LC3-II expression (Huang, 2016). These findings suggest that sphingolipids may play a role in Salmonellainduced cellular autophagy of damaged SCVs.

The cell ubiquitinates SCVs to trigger nuclear factor$\kappa \mathrm{B}(\mathrm{NF}-\kappa \mathrm{B})$ activation, which can lead to a reduction of bacterial propagation by the induction of inflammation. Furthermore, Salmonella escaping to the cytosol are tagged by a dense polyubiquitin coat (Narayanan and Edelmann, 2014). Both ceramide and NSM2 are connected to the regulation or modulation of protein ubiquitination and subsequent degradation (Chapman et al., 2005; Dobierzewska et al., 2011). Taking these facts into consideration, it is possible that sphingolipids play a role in the ubiquitination of the SCV.

\section{BETTER SAFE THAN SORRY - SPHINGOLIPIDS AS ANTIMICROBIALS}

Sphingolipids and sphingolipid signaling contribute to the immune response upon bacterial infection. Acid sphingomyelinase and ceramide play a decisive role in bacterial internalization and inflammatory response (Li et al., 2019). Sphingolipids, however, can also be applied as antimicrobial substances, regulating the growth and propagation of bacteria.

Around 30 years ago Bibel and colleagues pioneered the investigation of antimicrobial effects of sphingolipids. They reported that sphinganine influences the growth of $N$. meningitidis and Acinetobacter lwoffii and damages the cell wall of S. aureus (Bibel et al., 1993). Moreover, they tested sphinganine on human volunteers as a preventative antiseptic against subsequently applied $S$. aureus and observed an up to three-log reduction in the population of target micro-organisms compared to untreated controls (Bibel et al., 1995). Furthermore, it was shown that sphingosine effectively killed S. aureus, Streptococcus pyogenes, Micrococcus luteus, 
Propionibacterium acnes, Staphylococcus epidermidis and moderately killed $P$. aeruginosa. However, sphingosine was demonstrated to not influence the growth of E. coli and Serratia marcescens (Bibel et al., 1992). Although more research is needed to understand these differences in the effect of sphingosine, one can speculate that it might be related to the fact that E. coli and S. marcescens are Gram-negative enterobacteria, as opposed to the others, which are Gram-positive.

The increasing lack of antibiotic treatments due to the development of resistances demands novel approaches for therapies. A better understanding of the antibacterial effect of sphingolipids may offer novel targets for treatment. Within the last decade, the number of publications reporting antimicrobial effects of different derivatives of sphingolipids has increased.

For instance, Banhart et al. (2014) demonstrated that the sphingomyelin synthase inhibitor D609, which has an effect on the uptake of fluorescently labeled ceramide by Chlamydia muridarum (Elwell et al., 2011), reduces the propagation of C. trachomatis. To better understand the impact of sphingomyelin production on the growth of C. trachomatis, they synthesized several ceramide derivatives, such as nitrobenzooxadiazole (NBD)-labeled 1-O-methylceramide- $\mathrm{C}_{16}$. This derivative resembles to a large extent a compound called 1-O-methyl- $\mathrm{C}_{6}-\mathrm{NBD}$-ceramide, which has been shown not to be converted to sphingomyelin. Interestingly, the treatment with this newly synthetized ceramide inhibits chlamydial growth similar to chloramphenicol and 17 times more effectively than D609 (Banhart et al., 2014).

In another study, the antibacterial activity of sphingosine, as well as short-chain C6 and long-chain C16-ceramides and azido functionalized ceramide analogs was tested. The study revealed that short-chain ceramides and a $\omega$-azido-C6-ceramide had antibacterial effects on $N$. meningitidis and N. gonorrhoeae. The uptake of ceramides by Neisseria happened rapidly within $5 \mathrm{~min}$, and the killing occurred within $2 \mathrm{~h}$. In contrast to Neisseria, these analogs did not display any effects on E. coli and S. aureus (Becam et al., 2017). However, E. coli and $S$. aureus were shown to be efficiently killed by the treatment of dihydrosphingosine and sphingosine (Fischer et al., 2013). During $P$. aeruginosa infection, lower levels of sphingosine were observed due to a reduced activity of acid ceramidase, catalyzing the reaction of ceramide to sphingosine. By normalization of sphingosine levels, the susceptibility to $P$. aeruginosa could be decreased (Pewzner-Jung et al., 2014). Sphingosine, sphinganine and phytosphingosine were demonstrated to have a strong effect on biofilm formation and adherence of Streptococcus mutans (Cukkemane et al., 2015; Figure 2).

Beside host sphingolipids or chemically synthesized sphingolipids, extracted sphingolipids of plants have antibacterial

\section{REFERENCES}

Agaisse, H., and Derre, I. (2014). Expression of the effector protein IncD in Chlamydia trachomatis mediates recruitment of the lipid transfer protein CERT and the endoplasmic reticulum-resident protein VAPB to the inclusion membrane. Infect. Immun. 82, 2037-2047. doi: 10.1128/IAI.01530- 14 activity, as well. For example, the sphingolipids ficusamide, $(\mathrm{S})-(-)$ oxypeucedanin hydrate and (R)- $(+)$ oxypeucedanin hydrate of Ficus exasperata showed antibacterial activity. While ficusamide had only a low activity against E. coli, $(\mathrm{S})-(-)$ oxypeucedanin hydrate and (R)-(+) oxypeucedanin hydrate showed significant activity against Bacillus cereus (Dongfack et al., 2012).

\section{CONCLUDING REMARKS}

In the recent years, we have become increasingly aware of the importance of lipids, and sphingolipids in particular, for the processes of infection with and defense against pathogenic bacteria. Bacterial invasion or uptake are often mediated by bacterial attachment to glycosphingolipids or regulated by the increase in plasma membrane ceramide. Bacterial internalization can be a mechanism that increases pathogen survival, but sometimes it is also a part of the immunity, where again sphingolipids participate in the destruction of bacteria through regulating phagosome/lysosome fusion, apoptosis, or the inflammatory response. Intracellular bacteria face the challenges of survival within the cell and here also sphingolipids can be a tool to control autophagy and enable survival, or serve as building blocks for bacterial inclusions, ensuring their reproduction. However, sphingolipids can be applied as antimicrobials, as well, negatively influencing bacterial growth and biofilm formation. Understanding exact mechanisms behind these processes remains a challenge for the future and, in the wake of increasing antibiotic resistance, will be of great value in our fight against bacterial pathogens.

\section{AUTHOR CONTRIBUTIONS}

TK and VK-P wrote the manuscript and prepared the figures.

\section{FUNDING}

This publication was funded by the German Research Foundation (DFG) and the University of Würzburg in the funding program Open Access Publishing.

\section{ACKNOWLEDGMENTS}

We thank David Krones for critically reading the manuscript.

Bajwa, A., Rosin, D. L., Chroscicki, P., Lee, S., Dondeti, K., Ye, H., et al. (2015) Sphingosine 1-phosphate receptor-1 enhances mitochondrial function and reduces cisplatin-induced tubule injury. J. Am. Soc. Nephrol. 26, 908-925. doi: 10.1681/ASN.2013121351

Banhart, S., Saied, E. M., Martini, A., Koch, S., Aeberhard, L., Madela, K., et al. (2014). Improved plaque assay identifies a novel anti-Chlamydia ceramide 
derivative with altered intracellular localization. Antimicrob. Agents Chemother 58, 5537-5546. doi: 10.1128/AAC.03457- 14

Becam, J., Walter, T., Burgert, A., Schlegel, J., Sauer, M., Seibel, J., et al. (2017). Antibacterial activity of ceramide and ceramide analogs against pathogenic Neisseria. Sci. Rep. 7:17627. doi: 10.1038/s41598-017-18 071-w

Becker, K. A., Fahsel, B., Kemper, H., Mayeres, J., Li, C., Wilker, B., et al. (2017). Staphylococcus aureus alpha-toxin disrupts endothelial-cell tight junctions via acid sphingomyelinase and ceramide. Infect. Immun. 86:e00606-17. doi: 10. 1128/IAI.00606-17

Bibel, D. J., Aly, R., Shah, S., and Shinefield, H. R. (1993). Sphingosines: antimicrobial barriers of the skin. Acta Derm. Venereol. 73, 407-411.

Bibel, D. J., Aly, R., and Shinefield, H. R. (1992). Antimicrobial activity of sphingosines. J. Invest. Dermatol. 98, 269-273.

Bibel, D. J., Aly, R., and Shinefield, H. R. (1995). Topical sphingolipids in antisepsis and antifungal therapy. Clin. Exp. Dermatol 20, 395-400. doi: 10.1111/j.13652230.1995.tb01356.x

Capmany, A., and Damiani, M. T. (2010). Chlamydia trachomatis intercepts Golgi-derived sphingolipids through a Rab14-mediated transport required for bacterial development and replication. PLoS One 5:e14084. doi: 10.1371/ journal.pone. 0014084

Capmany, A., Gambarte Tudela, J., Alonso Bivou, M., and Damiani, M. T. (2019). Akt/AS160 signaling pathway inhibition impairs infection by decreasing Rab14-controlled sphingolipids delivery to chlamydial inclusions. Front. Microbiol. 10:666. doi: 10.3389/fmicb.2019.00666

Castro-Garza, J., González-Salazar, F., Quinn, F. D., Karls, R. K., De La Garza-Salinas, L. H., Guzmán-de la Garza, F. J., et al. (2016). An acidic sphingomyelinase Type C activity from Mycobacterium tuberculosis. Rev. Argent. Microbiol. 48, 21-26. doi: 10.1016/j.ram.2016.01.001

Cazalet, C., Rusniok, C., Bruggemann, H., Zidane, N., Magnier, A., Ma, L., et al. (2004). Evidence in the Legionella pneumophila genome for exploitation of host cell functions and high genome plasticity. Nat. Genet. 36, 1165-1173. doi: $10.1038 /$ ng 1447

Chapman, H., Ramstrom, C., Korhonen, L., Laine, M., Wann, K. T., Lindholm, D., et al. (2005). Downregulation of the HERG (KCNH2) $\mathrm{K}(+)$ channel by ceramide: evidence for ubiquitin-mediated lysosomal degradation. J. Cell Sci. 118, 5325-5334. doi: $10.1242 /$ jcs. 02635

Cukkemane, N., Bikker, F. J., Nazmi, K., Brand, H. S., Sotres, J., Lindh, L., et al. (2015). Anti-adherence and bactericidal activity of sphingolipids against Streptococcus mutans. Eur. J. Oral Sci. 123, 221-227. doi: 10.1111/eos.12200

Cuvillier, O., Pirianov, G., Kleuser, B., Vanek, P. G., Coso, O. A., Gutkind, J. S., et al. (1996). Suppression of ceramide-mediated programmed cell death by sphingosine-1-phosphate. Nature 381, 800-803. doi: 10.1038/381800a0

Davaille, J., Gallois, C., Habib, A., Li, L., Mallat, A., Tao, J., et al. (2000). Antiproliferative properties of sphingosine 1-phosphate in human hepatic myofibroblasts: a cyclooxygenase-2 mediated pathway. J. Biol. Chem. 275, 34628-34633. doi: 10.1074/jbc.m006393200

Deretic, V., Saitoh, T., and Akira, S. (2013). Autophagy in infection, inflammation and immunity. Nat. Rev. Immunol. 13, 722-737. doi: 10.1038/nri3532

Dobierzewska, A., Giltiay, N. V., Sabapathi, S., Karakashian, A. A., and Nikolova-Karakashian, M. N. (2011). Protein phosphatase 2A and neutral sphingomyelinase 2 regulate IRAK-1 protein ubiquitination and degradation in response to interleukin-1beta. J. Biol. Chem. 286, 32064-32073. doi: 10.1074/ jbc.M111.238030

Dongfack, M. D., Lallemand, M. C., Kuete, V., Mbazoa, C. D., Wansi, J. D., Trinhvan-Dufat, H., et al. (2012). A new sphingolipid and furanocoumarins with antimicrobial activity from Ficus exasperata. Chem. Pharm. Bull. 60, 1072-1075. doi: $10.1248 / \mathrm{cpb} . c 12-00279$

Ebenezer, D. L., Berdyshev, E. V., Bronova, I. A., Liu, Y., Tiruppathi, C., Komarova, Y., et al. (2019). Pseudomonas aeruginosa stimulates nuclear sphingosine-1phosphate generation and epigenetic regulation of lung inflammatory injury. Thorax 74, 579-591. doi: 10.1136/thoraxjnl-2018-212378

Ellington, A. A., Berhow, M. A., and Singletary, K. W. (2006). Inhibition of Akt signaling and enhanced ERK1/2 activity are involved in induction of macroautophagy by triterpenoid B-group soyasaponins in colon cancer cells. Carcinogenesis 27, 298-306. doi: 10.1093/carcin/bgi214

Elwell, C. A., Jiang, S., Kim, J. H., Lee, A., Wittmann, T., Hanada, K., et al. (2011). Chlamydia trachomatis co-opts GBF1 and CERT to acquire host sphingomyelin for distinct roles during intracellular development. PLoS Pathog. 7:e1002198. doi: 10.1371/journal.ppat.1002198

Faulstich, M., Hagen, F., Avota, E., Kozjak-Pavlovic, V., Winkler, A.-C., Xian, Y., et al. (2015). Neutral sphingomyelinase 2 is a key factor for PorB-dependent invasion of Neisseria gonorrhoeae. Cell Microbiol. 17, 241-253. doi: 10.1111/cmi. 12361

Fischer, C. L., Walters, K. S., Drake, D. R., Blanchette, D. R., Dawson, D. V., Brogden, K. A., et al. (2013). Sphingoid bases are taken up by Escherichia coli and Staphylococcus aureus and induce ultrastructural damage. Skin Pharmacol. Physiol. 26, 36-44. doi: 10.1159/000343175

Gault, C. R., Obeid, L. M., and Hannun, Y. A. (2010). An overview of sphingolipid metabolism: from synthesis to breakdown. Adv. Exp. Med. Biol. 688, 1-23. doi: 10.1007/978-1-4419-6741-1_1

Gillard, B. K., Clement, R. G., and Marcus, D. M. (1998). Variations among cell lines in the synthesis of sphingolipids in de novo and recycling pathways. Glycobiology 8, 885-890. doi: 10.1093/glycob/8.9.885

Gomez-Valero, L., and Buchrieser, C. (2013). Genome dynamics in Legionella: the basis of versatility and adaptation to intracellular replication. Cold Spring Harb. Perspect. Med. 3:a009993. doi: 10.1101/cshperspect.a009993

Grassmé, H., Gulbins, E., Brenner, B., Ferlinz, K., Sandhoff, K., Harzer, K., et al. (1997). Acidic sphingomyelinase mediates entry of N. gonorrhoeae into nonphagocytic cells. Cell 91, 605-615. doi: 10.1016/s0092-8674(00)80448-1

Grassme, H., Jendrossek, V., Riehle, A., von Kurthy, G., Berger, J., Schwarz, H., et al. (2003). Host defense against Pseudomonas aeruginosa requires ceramide-rich membrane rafts. Nat. Med. 9, 322-330. doi: 10.1038/nm823

Grösch, S., Schiffmann, S., and Geisslinger, G. (2012). Chain length-specific properties of ceramides. Prog. Lipid Res. 51, 50-62. doi: 10.1016/j.plipres.2011. 11.001

Hackstadt, T., Scidmore, M. A., and Rockey, D. D. (1995). Lipid metabolism in Chlamydia trachomatis-infected cells: directed trafficking of Golgi-derived sphingolipids to the chlamydial inclusion. Proc. Natl. Acad. Sci. U.S.A. 92, 4877-4881. doi: 10.1073/pnas.92.11.4877

Hanada, K. (2005). Sphingolipids in infectious diseases. Jpn. J. Infect. Dis. 58, $131-148$.

Harvald, E. B., Olsen, A. S. B., and Faergeman, N. J. (2015). Autophagy in the light of sphingolipid metabolism. Apoptosis 20, 658-670. doi: 10.1007/s10495-0151108-2

Harvey, H. A., Swords, W. E., and Apicella, M. A. (2001). The mimicry of human glycolipids and glycosphingolipids by the lipooligosaccharides of pathogenic Neisseria and Haemophilus. J. Autoimmun. 16, 257-262. doi: 10.1006/jaut.2000. 0477

Hauck, C. R., Grassmé, H., Bock, J., Jendrossek, V., Ferlinz, K., Meyer, T. F., et al. (2000). Acid sphingomyelinase is involved in CEACAM receptor-mediated phagocytosis of Neisseria gonorrhoeae. FEBS Lett. 478, 260-266. doi: 10.1016/ s0014-5793(00)01851-2

Hernández-Corbacho, M. J., Salama, M. F., Canals, D., Senkal, C. E., and Obeid, L. M. (2017). Sphingolipids in mitochondria. Biochim. Biophys. Acta Mol. Cell Biol. Lipids 1862, 56-68.

Herweg, J. A., Pons, V., Becher, D., Hecker, M., Krohne, G., Barbier, J., et al. (2016). Proteomic analysis of the Simkania-containing vacuole: the central role of retrograde transport. Mol. Microbiol. 99, 151-171. doi: 10.1111/mmi.13222

Heuer, D., Rejman Lipinski, A., Machuy, N., Karlas, A., Wehrens, A., Siedler, F., et al. (2009). Chlamydia causes fragmentation of the Golgi compartment to ensure reproduction. Nature 457, 731-735. doi: 10.1038/nature07578

Holmgren, J., Lönnroth, I., Månsson, J., and Svennerholm, L. (1975). Interaction of cholera toxin and membrane GM1 ganglioside of small intestine. Proc. Natl. Acad. Sci. U.S.A. 72, 2520-2524. doi: 10.1073/pnas.72.7.2520

Huang, F. C. (2016). De Novo sphingolipid synthesis is essential for Salmonellainduced autophagy and human beta-defensin 2 expression in intestinal epithelial cells. Gut. Pathog. 8:5. doi: 10.1186/s13099-016-0088-2

Huang, F. C. (2017). The role of sphingolipids on innate immunity to intestinal Salmonella Infection. Int. J. Mol. Sci. 18:1720. doi: 10.3390/ijms18081720

Huang, J., and Brumell, J. H. (2014). Bacteria-autophagy interplay: a battle for survival. Nat. Rev. Microbiol. 12, 101-114. doi: 10.1038/nrmicro 3160

Hugosson, S., Angström, J., Olsson, B.-M., Bergströmlm, J., Fredlund, H., Olcen, P., et al. (1998). Glycosphingolipid binding specificities of Neisseria meningitidis and Haemophilus influenzae: detection, isolation, and characterization of 
a binding-active glycosphingolipid from human oropharyngeal epithelium. J. Biochem. 124, 1138-1152. doi: 10.1093/oxfordjournals.jbchem.a022232

James, P. F., and Zoeller, R. A. (1997). Isolation of animal cell mutants defective in long-chain fatty aldehyde dehydrogenase: sensitivity to fatty aldehydes and Schiff's base modification of phospholipids: implications for Sjörgen-Larsson syndrome. J. Biol. Chem. 272, 23532-23539. doi: 10.1074/jbc.272.38.23532

Kanazawa, T., Taneike, I., Akaishi, R., Yoshizawa, F., Furuya, N., Fujimura, S., et al. (2004). Amino acids and insulin control autophagic proteolysis through different signaling pathways in relation to mTOR in isolated rat hepatocytes. J. Biol. Chem. 279, 8452-8459. doi: 10.1074/jbc.m306337200

Kavaliauskiene, S., Dyve Lingelem, A. B., Skotland, T., and Sandvig, K. (2017). Protection against shiga toxins. Toxins 9:e44.

Koch-Edelmann, S., Banhart, S., Saied, E. M., Rose, L., Aeberhard, L., Laue, M., et al. (2017). The cellular ceramide transport protein CERT promotes Chlamydia psittaci infection and controls bacterial sphingolipid uptake. Cell Microbiol. 19:e12752. doi: 10.1111/cmi.12752

Kolter, T., and Sandhoff, K. (2005). Principles of lysosomal membrane digestion: stimulation of sphingolipid degradation by sphingolipid activator proteins and anionic lysosomal lipids. Annu. Rev. Cell Dev. Biol. 21, 81-103. doi: 10.1146/ annurev.cellbio.21.122303.120013

Kühlewein, C., Rechner, C., Meyer, T. F., and Rudel, T. (2006). Low-phosphatedependent invasion resembles a general way for Neisseria gonorrhoeae to enter host cells. Infect. Immun. 74, 4266-4273. doi: 10.1128/iai.00215-06

Lépine, S., Allegood, J. C., Park, M., Dent, P., Milstien, S., and Spiegel, S. (2011). Sphingosine-1-phosphate phosphohydrolase-1 regulates ER stressinduced autophagy. Cell Death Differ. 18, 350-361. doi: 10.1038/cdd. 2010.104

Li, C., Wang, A., Wu, Y., Gulbins, E., Grassme, H., and Zhao, Z. (2019). Acid sphingomyelinase-ceramide system in bacterial infections. Cell Physiol. Biochem 52, 280-301. doi: 10.33594/000000021

Liu, H., Toman, R. E., Goparaju, S. K., Maceyka, M., Nava, V. E., Sankala, H., et al. (2003). sphingosine kinase type 2 is a putative BH3-only protein that induces apoptosis. J. Biol. Chem. 278, 40330-40336. doi: 10.1074/jbc.m304455200

Liu, Y., Yang, Y., Ye, Y. C., Shi, Q. F., Chai, K., Tashiro, S., et al. (2012). Activation of ERK-p53 and ERK-mediated phosphorylation of Bcl-2 are involved in autophagic cell death induced by the c-Met inhibitor SU11274 in human lung cancer A549 cells. J. Pharmacol. Sci. 118, 423-432. doi: 10.1254/jphs.11181fp

Ma, J., Gulbins, E., Edwards, M. J., Caldwell, C. C., Fraunholz, M., and Becker, K. A. (2017). Staphylococcus aureus $\alpha$-Toxin induces inflammatory cytokines via lysosomal acid sphingomyelinase and ceramides. Cell Physiol. Biochem. 43, 2170-2184. doi: 10.1159/000484296

Managò, A., Becker, K. A., Carpinteiro, A., Wilker, B., Soddemann, M., Seitz, A. P., et al. (2015). Pseudomonas aeruginosa pyocyanin induces neutrophil death via mitochondrial reactive oxygen species and mitochondrial acid sphingomyelinase. Antioxid. Redox. Signal. 22, 1097-1110. doi: 10.1089/ars. 2014.5979

Menaldino, D. S., Bushnev, A., Sun, A., Liotta, D. C., Symolon, H., Desai, K., et al. (2003). Sphingoid bases and de novo ceramide synthesis: enzymes involved, pharmacology and mechanisms of action. Pharmacol. Res. 47, 373-381. doi: 10.1016/s1043-6618(03)00054-9

Mencarelli, C., and Martinez-Martinez, P. (2013). Ceramide function in the brain: when a slight tilt is enough. Cell Mol. Life Sci. 70, 181-203. doi: 10.1007/s00018012-1038-x

Miller, C., and Celli, J. (2016). Avoidance and subversion of eukaryotic homeostatic autophagy mechanisms by bacterial pathogens. J. Mol. Biol. 428, 3387-3398. doi: 10.1016/j.jmb.2016.07.007

Mital, J., and Hackstadt, T. (2011). Role for the SRC family kinase Fyn in sphingolipid acquisition by chlamydiae. Infect. Immun. 79, 4559-4568. doi: 10.1128/IAI.05692-11

Moore, E. R., Mead, D. J., Dooley, C. A., Sager, J., and Hackstadt, T. (2011). The trans-Golgi SNARE syntaxin 6 is recruited to the chlamydial inclusion membrane. Microbiology 157, 830-838. doi: 10.1099/mic.0.045856-0

Narayanan, L. A., and Edelmann, M. J. (2014). Ubiquitination as an efficient molecular strategy employed in Salmonella infection. Front Immunol 5:558. doi: 10.3389/fimmu.2014.00558

Newton, H. J., Ang, D. K., van Driel, I. R., and Hartland, E. L. (2010). Molecular pathogenesis of infections caused by Legionella pneumophila. Clin. Microbiol. Rev. 23, 274-298. doi: 10.1128/CMR.00052-09
Newton, J., Lima, S., Maceyka, M., and Spiegel, S. (2015). Revisiting the sphingolipid rheostat: evolving concepts in cancer therapy. Exp. Cell Res. 333, 195-200. doi: 10.1016/j.yexcr.2015.02.025

Ogier-Denis, E., Pattingre, S., El Benna, J., and Codogno, P. (2000). Erk1/2dependent phosphorylation of Galpha-interacting protein stimulates its GTPase accelerating activity and autophagy in human colon cancer cells. J. Biol. Chem. 275, 39090-39095. doi: 10.1074/jbc.m006198200

Okino, N., and Ito, M. (2007). Ceramidase enhances phospholipase C-induced hemolysis by Pseudomonas aeruginosa. J. Biol. Chem. 282, 6021-6030. doi: 10.1074/jbc.m603088200

Okino, N., Tani, M., Imayama, S., and Ito, M. (1998). Purification and characterization of a novel ceramidase from Pseudomonas aeruginosa. J. Biol. Chem. 273, 14368-14373. doi: 10.1074/jbc.273.23.14368

Owen, K. A., Meyer, C. B., Bouton, A. H., and Casanova, J. E. (2014). Activation of focal adhesion kinase by Salmonella suppresses autophagy via an Akt/mTOR signaling pathway and promotes bacterial survival in macrophages. PLoS Pathog. 10:e1004159. doi: 10.1371/journal.ppat.1004159

Peng, H., Li, C., Kadow, S., Henry, B. D., Steinmann, J., Becker, K. A., et al. (2015). Acid sphingomyelinase inhibition protects mice from lung edema and lethal Staphylococcus aureus sepsis. J. Mol. Med. 93, 675-689. doi: 10.1007/s00109014-1246-y

Perry, D. K. (2002). Serine palmitoyltransferase: role in apoptotic de novo ceramide synthesis and other stress responses. Biochim. Biophys. Acta 1585, 146-152. doi: 10.1016/s1388-1981(02)00335-9

Peters, S., Schlegel, J., Becam, J., Avota, E., Sauer, M., and Schubert-Unkmeir, A. (2019). Neisseria meningitidis type IV pili trigger Ca2+-dependent lysosomal trafficking of the acid sphingomyelinase to enhance surface ceramide levels. Infect. Immun. 87:e0410-19. doi: 10.1128/IAI.00410-19

Pewzner-Jung, Y., Tavakoli Tabazavareh, S., Grassme, H., Becker, K. A., Japtok, L., Steinmann, J., et al. (2014). Sphingoid long chain bases prevent lung infection by Pseudomonas aeruginosa. EMBO Mol Med 6, 1205-1214. doi: 10.15252/emmm. 201404075

Riboni, L., Viani, P., Bassi, R., Prinetti, A., and Tettamanti, G. (1997). The role of sphingolipids in the process of signal transduction. Prog. Lipid Res. 36, 153-195. doi: 10.1016/s0163-7827(97)00008-8

Rolando, M., Escoll, P., and Buchrieser, C. (2016a). Legionella pneumophila restrains autophagy by modulating the host's sphingolipid metabolism. Autophagy 12, 1053-1054. doi: 10.1080/15548627.2016.1166325

Rolando, M., Escoll, P., Nora, T., Botti, J., Boitez, V., Bedia, C., et al. (2016b). Legionella pneumophila S1P-lyase targets host sphingolipid metabolism and restrains autophagy. Proc. Natl. Acad. Sci. U.S.A. 113, 1901-1906. doi: 10.1073/ pnas. 1522067113

Rudel, T., Kepp, O., and Kozjak-Pavlovic, V. (2010). Interactions between bacterial pathogens and mitochondrial cell death pathways. Nat. Rev. Microbiol. 8, 693-705. doi: 10.1038/nrmicro2421

Saiman, L., and Prince, A. (1993). Pseudomonas aeruginosa pili bind to asialoGM1 which is increased on the surface of cystic fibrosis epithelial cells. J. Clin. Invest. 92, 1875-1880. doi: 10.1172/jci116779

Sarkar, S., Maceyka, M., Hait, N. C., Paugh, S. W., Sankala, H., Milstien, S., et al. (2005). Sphingosine kinase 1 is required for migration, proliferation and survival of MCF-7 human breast cancer cells. FEBS Lett. 579, 5313-5317. doi: 10.1016/j.febslet.2005.08.055

Scarlatti, F., Bauvy, C., Ventruti, A., Sala, G., Cluzeaud, F., Vandewalle, A., et al. (2004). Ceramide-mediated macroautophagy involves inhibition of protein kinase B and up-regulation of beclin 1. J. Biol. Chem. 279, 18384-18391. doi: 10.1074/jbc.m313561200

Simonis, A., Hebling, S., Gulbins, E., Schneider-Schaulies, S., and SchubertUnkmeir, A. (2014). Differential activation of acid sphingomyelinase and ceramide release determines invasiveness of Neisseria meningitidis into brain endothelial cells. PLoS Pathog. 10:e1004160. doi: 10.1371/journal.ppat.1004160

Simonis, A., and Schubert-Unkmeir, A. (2018). The role of acid sphingomyelinase and modulation of sphingolipid metabolism in bacterial infection. Biol. Chem. 399, 1135-1146. doi: 10.1515/hsz-2018-0200

Simons, K., and Ikonen, E. (1997). Functional rafts in cell membranes. Nature 387, 569-572. doi: 10.1038/42408

Siqueira, M. D. S., Ribeiro, R. M., and Travassos, L. H. (2018). Autophagy and its interaction with intracellular bacterial pathogens. Front. Immunol. 9:935. doi: 10.3389/fimmu.2018.00935 
Speer, A., Sun, J., Danilchanka, O., Meikle, V., Rowland, J. L., Walter, K., et al. (2015). Surface hydrolysis of sphingomyelin by the outer membrane protein Rv0888 supports replication of Mycobacterium tuberculosis in macrophages. Mol. Microbiol. 97, 881-897. doi: 10.1111/mmi. 13073

Taniguchi, M., Kitatani, K., Kondo, T., Hashimoto-Nishimura, M., Asano, S., Hayashi, A., et al. (2012). Regulation of autophagy and its associated cell death by "sphingolipid rheostat": reciprocal role of ceramide and sphingosine 1-phosphate in the mammalian target of rapamycin pathway. J. Biol. Chem. 287, 39898-39910. doi: 10.1074/jbc.M112.41 6552

Tattoli, I., Sorbara, M. T., Vuckovic, D., Ling, A., Soares, F., Carneiro, L. A., et al. (2012). Amino acid starvation induced by invasive bacterial pathogens triggers an innate host defense program. Cell Host Microbe 11, 563-575. doi: 10.1016/j.chom.2012.04.012

Tettamanti, G., Bassi, R., Viani, P., and Riboni, L. (2003). Salvage pathways in glycosphingolipid metabolism. Biochimie 85, 423-437. doi: 10.1016/s03009084(03)00047-6

van Ooij, C., Kalman, L., van, I., Nishijima, M., Hanada, K., Mostov, K., et al. (2000). Host cell-derived sphingolipids are required for the intracellular growth of Chlamydia trachomatis. Cell Microbiol. 2, 627-637. doi: 10.1046/j.1462-5822. 2000.00077.x

Viswanathan, G., Jafurulla, M., Kumar, G. A., Raghunand, T. R., and Chattopadhyay, A. (2018). Macrophage sphingolipids are essential for the entry of mycobacteria. Chem. Phys. Lipids 213, 25-31. doi: 10.1016/j.chemphyslip. 2018.03.004
Wolf, K., and Hackstadt, T. (2001). Sphingomyelin trafficking in Chlamydia pneumoniae-infected cells. Cell Microbiol. 3, 145-152. doi: 10.1046/j.14625822.2001.00098.x

Young, M. M., Kester, M., and Wang, H. G. (2013). Sphingolipids: regulators of crosstalk between apoptosis and autophagy. J. Lipid Res. 54, 5-19. doi: 10.1194/ jlr.R031278

Young, M. M., and Wang, H. G. (2018). Sphingolipids as regulators of autophagy and endocytic trafficking. Adv. Cancer Res. 140, 27-60. doi: 10.1016/bs.acr.2018. 04.008

Zhang, Y., Li, X., Carpinteiro, A., and Gulbins, E. (2008). Acid sphingomyelinase amplifies redox signaling in Pseudomonas aeruginosa-induced macrophage apoptosis. J. Immunol. 181, 4247-4254. doi: 10.4049/jimmunol.181.6.4247

Conflict of Interest: The authors declare that the research was conducted in the absence of any commercial or financial relationships that could be construed as a potential conflict of interest.

The reviewer PE declared a past supervisory role with one of the authors TK to the handling Editor.

Copyright (c) 2019 Kunz and Kozjak-Pavlovic. This is an open-access article distributed under the terms of the Creative Commons Attribution License (CC BY).

The use, distribution or reproduction in other forums is permitted, provided the original author(s) and the copyright owner(s) are credited and that the original publication in this journal is cited, in accordance with accepted academic practice. No use, distribution or reproduction is permitted which does not comply with these terms. 